\title{
Venta de alcohol y tasa de enfermedad hepática alcohólica por departamentos en Colombia
}

\section{Alcoholic Beverage Sales and Alcoholic Liver Disease Rates in Colombian Departments}

\author{
Valentina Andrade ${ }^{1}$, Juan David Mosos ${ }^{1}$, Bethia Pacheco ${ }^{1}$, Maria José Polanía', Daniela Yucumá1, Diego Rosselli, MD²
}

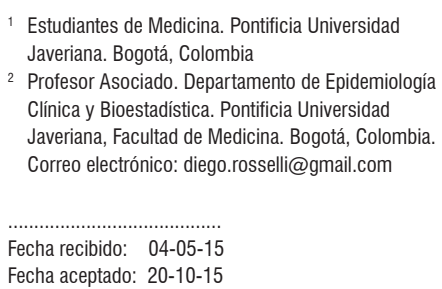

\begin{abstract}
Resumen
Objetivos: determinar la asociación estadística entre el diagnóstico de enfermedad hepática alcohólica según Registros Individuales de Prestaciones de Servicios de Salud (RIPS) y ventas de alcohol por departamento, 2012. Métodos: se realizó un estudio ecológico transversal, comparando tasas de prevalencia estimadas por RIPS (códigos CIE K700-9) y población mayor de 18 años (DANE), con ventas de licores nacionales, licores importados y cervezas, convertidas a unidades de alcohol, según datos de la Federación Nacional de Departamentos. Resultados: las tasa de enfermedad hepática alcohólica nacional fue 10,7 por 100000 con las tasas más elevadas en Santander $(27,1)$, Risaralda $(19,9)$ y Boyacá $(15,0)$. Después de retirar los datos de departamentos con resultados poco confiables o incompletos, la correlación de Pearson para tasa de enfermedad y ventas de unidades de alcohol fue de 0,6 . Conclusiones: existe una correlación positiva entre la tasa de enfermedad hepática alcohólica y las ventas de alcohol por departamento.
\end{abstract}

\section{Palabras clave}

Cirrosis hepática, enfermedad hepática alcohólica, bebidas alcohólicas, sistema de registros.

\begin{abstract}
Objective: The purpose of this study was to determine the statistical association between the diagnosis of alcoholic liver disease and alcohol sales by department based on the Individual Records of the Health Care Services (Registros Individuales de Prestaciones de Servicios de Salud - RIPS) for 2012. Methods: This was a cross-sectional ecologic study conducted to compare prevalence rates of alcoholic liver disease estimated by RIPS (ICD K700-9) in the over 18 population (DANE) with sales, according to the National Federation of Departments, of domestic and imported spirits, liquors and beers, converted into alcohol units. Results: The national rate of alcoholic liver disease was 10.7 per 100,000 with higher rates in Santander (27.1), Risaralda (19.9) and Boyacá (15.0). After removing the data from departments with unreliable or incomplete results, the Pearson correlation rate of illness and alcohol unit sales was 0.6 . Conclusions: There is a positive correlation between the rate of alcoholic liver disease and alcohol sales in each department.
\end{abstract}

\section{Keywords}

Liver cirrhosis, alcoholic liver disease, alcoholic beverages, record system.

\section{INTRODUCCIÓN}

Las consecuencias sobre el organismo del consumo crónico de alcohol son menos conocidas por el común de las personas que las consecuencias de la intoxicación aguda.
La razón, en parte, es que pueden pasar desapercibidas por un largo tiempo. Una de las alteraciones mejor conocida es la cirrosis hepática, cuya asociación con ingesta de alcohol, así como sus cambios histológicos, fueron hechos por Frank Burr Mallory en 1910 (1). El consumo de bebidas 
alcohólicas es parte integral del estilo de vida de muchos pueblos (2), llegando en ocasiones a ser más "normal y deseable" que el no consumo, en especial en el mundo occidental (3). Colombia no es la excepción en este contexto, pues el consumo de alcohol está ampliamente difundido en el país; las cifras señalan que entre $70 \%$ y $90 \%$ de la población lo ha probado (4). Esto implica que el consumo inadecuado de alcohol es un problema de relevancia social, cuyas consecuencias afectan ampliamente el fortalecimiento del capital humano y del capital social (5).

Los estudios colombianos sobre la asociación de enfermedad hepática con la ingesta de bebidas alcohólicas son pocos y de tipo anecdótico $(6,7)$. A la fecha no se han encontrado estudios publicados en Colombia que expongan tasas de enfermedad hepática y su correlación con el consumo de alcohol. El objetivo de este trabajo fue realizar un análisis relacional de los casos diagnosticados con enfermedad hepática alcohólica según los Registros Individuales de Prestaciones de Servicios de Salud (RIPS) y el consumo de alcohol por departamentos.

\section{MATERIAL Y MÉTODOS}

Esta investigación se considera como un estudio ecológico de grupos múltiples con carácter transversal. La unidad de análisis corresponde a una población geográficamente bien delimitada: habitantes colombianos con uno de los siguientes diagnósticos (códigos CIE-10) en los RIPS: cirrosis alcohólica (K703); hígado graso alcohólico (K700); hepatitis alcohólica (K701); fibrosis y esclerosis alcohólica del hígado (K702); fallo hepático alcohólico (K704) y enfermedad hepática alcohólica sin especificar (K709). La información se filtró por "diagnóstico principal" y "personas atendidas" y se escogió como año del análisis 2012 por ser el último con datos completos y por considerar la información de mejor calidad que la de los años precedentes $(8,9)$. Lamentablemente, los RIPS desconocen el método usado para llegar al diagnóstico.

Con información suministrada por la Federación Nacional de Departamentos -FND- (información no publicada) se obtuvieron datos de las cantidades estándares de licor (botellas de $750 \mathrm{~mL}$ para licor y $300 \mathrm{~mL}$ para cerveza) que pagaron impuestos por departamentos; se incluyen datos diferenciando licor nacional e importado; estos datos se convirtieron a unidades de alcohol, tomando cervezas con el $4 \%$ en volumen de alcohol y los licores con el $40 \%$. Para calcular la tasa de ventas per cápita y las tasas de enfermedad hepática alcohólica por departamento se utilizaron las proyecciones poblacionales del DANE por departamentos para individuos mayores de 18 años en el 2012. Las variables que se manejaron fueron edad, sexo, departamento en donde se prestó la atención en salud, y ventas de alcohol (que se interpretaron como una medida indirecta de consumo). Los 32 departamentos se tomaron como categorías (los datos de Bogotá se agruparon con los de Cundinamarca) y se estudiaron las otras variables por separado en cada uno.

\section{RESULTADOS}

En el año 2012 se atendieron en Colombia en total 3285 adultos ( 848 mujeres y 2437 hombres) con uno de los diagnósticos en estudio (cirrosis hepática alcohólica, hígado graso alcohólico, hepatitis alcohólica, fibrosis y esclerosis alcohólica del hígado, fallo hepático alcohólico y enfermedad hepática alcohólica sin especificar) para una tasa de 10,7 por 100000 habitantes. La mayor parte de estos pacientes fueron vistos en Cundinamarca (1046), Antioquia (451), Santander (387), Valle del Cauca (301) y Atlántico (160). Si se hubiera incluido en el análisis únicamente el diagnóstico de cirrosis hepática alcohólica, el total de pacientes habría sido de 2276 (443 mujeres y 1833 hombres).

La Tabla 1 muestra las ventas de alcohol por departamentos, en litros de alcohol per cápita, junto con nuestras tasas estimadas de enfermedad hepática alcohólica por cada 100000 habitantes, para 2012. La FND no tiene información de ventas de alcohol en Amazonas, Guaviare, Guainía, Putumayo, San Andrés, Vaupés y Vichada, por lo que se excluyeron del análisis. Los datos de Cundinamarca se manejan junto con los de Bogotá. Casanare tiene unos datos de ventas bastante atípicos, de dos y media veces el promedio nacional.

Los departamentos con mayores tasas de enfermedad hepática alcohólica por 100000 habitantes, para ambos sexos, fueron Santander $(27,1)$, Risaralda $(19,9)$ y Boyacá $(15,0)$. En mujeres, las tasas más elevadas se encontraron en Atlántico $(9,3)$ y Santander $(9,3)$. Por otro lado, los departamentos con tasas más bajas de enfermedad hepática alcohólica para ambos sexos fueron Putumayo $(0,52)$ y Chocó $(0,76)$. En el caso de las mujeres, las tasas más bajas corresponden a estos mismos departamentos, en donde no se registraron casos en mujeres en 2012.

Con respecto a las ventas per cápita de alcohol, los valores más altos, después de Casanare, se presentaron en Meta (6,21 litros de alcohol por habitante/año) seguido de cerca por Risaralda (5,68 litros). En los departamentos con los reportes más altos de venta de alcohol per cápita se encontró que estaba estrechamente relacionado con la tasa de enfermedad hepática alcohólica; en Cauca y Nariño donde la venta per cápita es menor, la tasa de enfermedad hepática alcohólica se comporta de la misma forma, teniendo estos departamentos unas tasas muy bajas, como se observa grá- 
ficamente en las Figuras 1 y 2. Al estimar un coeficiente de correlación de Pearson se obtiene como resultado 0,6.

Tabla 1. Ventas de alcohol per cápita y tasa de enfermedad hepática alcohólica por departamento en Colombia en el año 2012.

\begin{tabular}{lcc}
\hline \multicolumn{1}{c}{ Departamento } & $\begin{array}{c}\text { Venta de } \\
\text { alcohol per } \\
\text { cápita (litros) }\end{array}$ & $\begin{array}{c}\text { Tasas de } \\
\text { cirrosis (por } \\
100 \text { 000 } \\
\text { habitantes) }\end{array}$ \\
\hline Antioquia & 4,42 & 10,47 \\
Arauca & 2,78 & 9,93 \\
Atlántico & 3,49 & 10,05 \\
Bolívar & 4,43 & 5,45 \\
Boyacá & 3,84 & 15,04 \\
Caldas & 3,65 & 8,15 \\
Caquetá & 4,52 & 5,88 \\
Casanare & 10,32 & 8,14 \\
Cauca & 2,69 & 4,54 \\
Cesar & 2,89 & 3,75 \\
Chocó & 2,01 & 0,76 \\
Córdoba & 3,20 & 7,30 \\
Cundinamarca (con Bogotá) & 5,67 & 14,80 \\
Huila & 4,02 & 5,27 \\
La Guajira & 1,64 & 1,80 \\
Magdalena & 4,08 & 5,91 \\
Meta & 6,21 & 8,69 \\
Nariño & 2,30 & 4,61 \\
Norte de Santander & 4,00 & 10,51 \\
Quindío & 3,42 & 7,50 \\
Risaralda & 5,68 & 19,84 \\
Santander & 5,11 & 27,70 \\
Sucre & 3,28 & 10,03 \\
Tolima & 0,74 & \\
Valle del Cauca & 3,61 \\
Total nacional & & \\
\hline
\end{tabular}

En los departamentos de Antioquia, Atlántico, Caldas, Cauca, Córdoba, Nariño, Norte de Santander, Quindío y Valle del Cauca fueron en los que se evidenció una correlación más directa entre las ventas per cápita de alcohol y los casos diagnosticados en los RIPS para el 2012; en tanto que Santander, Boyacá, Risaralda y Tolima presentan muy altos reportes de enfermedad hepática alcohólica en los RIPS con relación a sus ventas de alcohol legales. El caso de Meta es atípico ya que presenta las ventas de alcohol per cápita más altas del territorio colombiano, sin embargo, las tasas de enfermedad hepática alcohólica en 2012 están por debajo del promedio nacional.

\section{DISCUSIÓN Y CONCLUSIONES}

Un estudio ecológico se basa en la observación de grupos y no en observaciones individuales, omitiendo características exclusivas de los sujetos (10). Por basarse en registro con potenciales problemas de subregistro (acentuados por el hecho de que muchos pacientes pueden negar el antecedente de ingesta de alcohol), de diagnósticos errados y otras posibles causas de error, este trabajo de investigación es susceptible de sesgo de información (11). La información tanto de los RIPS como de la Federación Nacional de Departamentos (que obviamente no incluye datos de contrabando) debe analizarse con cautela. Ni los pacientes que consultan a un lugar determinado necesariamente residen en ese departamento, ni el alcohol vendido se consume todo localmente. Además, nadie asegura que las personas consumidoras de alcohol sean las mismas con diagnóstico de cirrosis hepática alcohólica (10). Se podría agregar, como otra variable de confusión, que las tasas de cirrosis de hoy son más probablemente el reflejo del consumo de alcohol en el pasado (12), sumadas a factores genéticos y de estilo de vida $(13,14)$. Con el desarrollo de pruebas de laboratorio más específicas (15) la asociación entre alcohol y enfermedad hepática será más confiable.

A estas limitaciones de la información disponible se agregan algunos datos que son difíciles de interpretar, como ocurre con los departamentos de Casanare, Meta, Santander y Tolima (véase Tabla 1), que se desvían del comportamiento de los demás. En el caso de Santander se tendría una alta tasa de enfermedad hepática alcohólica con respecto a Meta y Casanare, por ejemplo, departamentos que lideran las ventas per cápita. Tolima, en cambio, tendría unas elevadas tasas de la enfermedad, a pesar de reportar unas ventas por debajo del promedio nacional. Las cifras de Casanare concuerdan con lo encontrado en la Encuesta Nacional de Demografía y Salud de Profamilia (16), donde las mujeres encuestadas en esta región oriental del país son las que reportan la más alta frecuencia de respuesta positiva a la pregunta de si su cónyuge se embriaga "alguna vez" $(35,2 \%)$ o "muchas veces" $(18,2 \%)$.

La exclusión de información del alcohol de contrabando (se estima que una de cada cuatro botellas consumidas en el país es de contrabando) afecta a los departamentos de manera diferente, lo que añade otro factor de imprecisión a nuestros resultados (17).

A pesar de las limitaciones expuestas, los resultados del trabajo muestran una correlación estadística entre el consumo de alcohol por departamento y el diagnóstico de enfermedad hepática alcohólica. Esto abre nuevas áreas potenciales de investigación, no solo para darle una mayor precisión a 


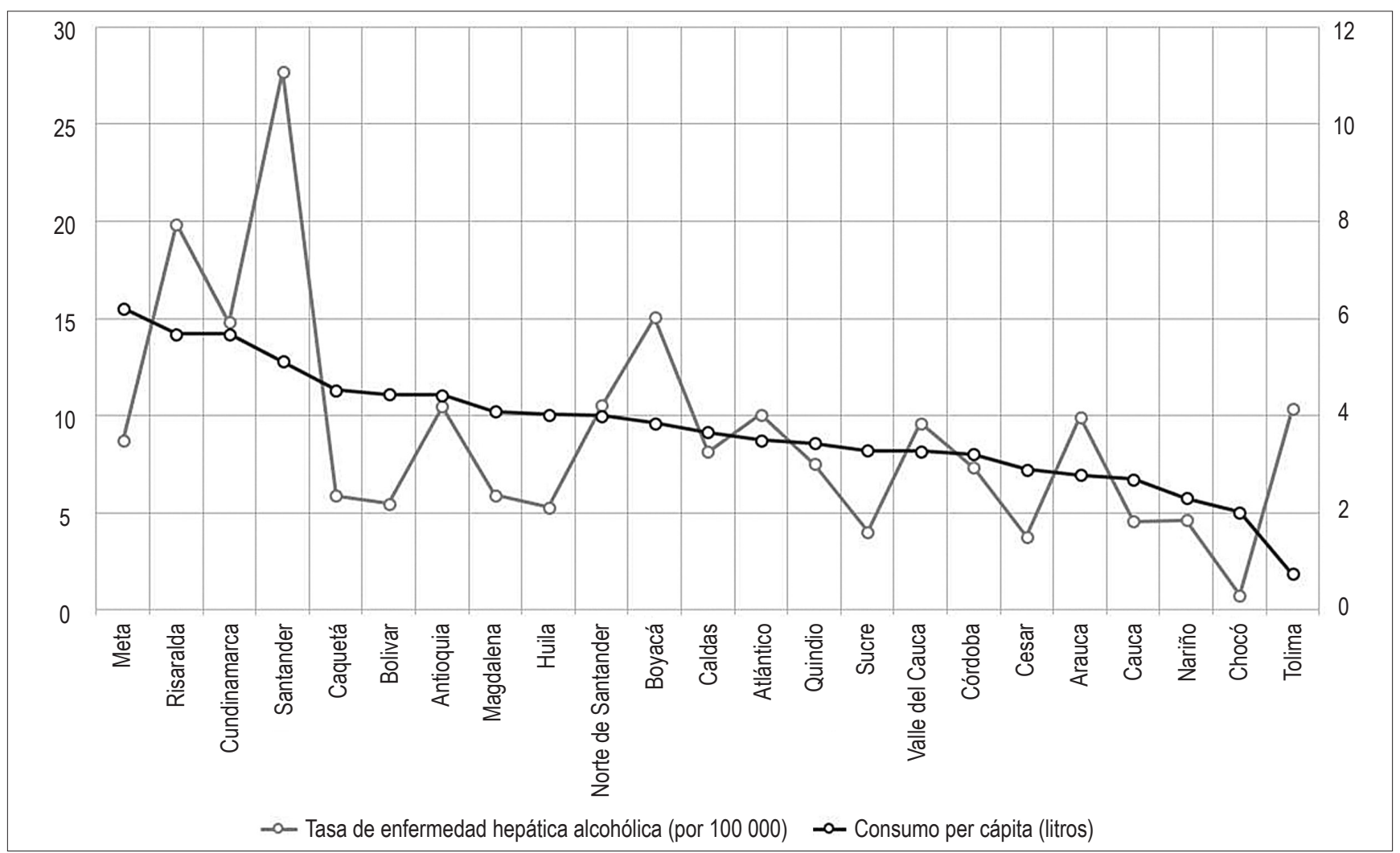

Figura 1. Comportamiento de la tasa de enfermedad hepática alcohólica y las ventas de alcohol per cápita por departamento en el año 2012.

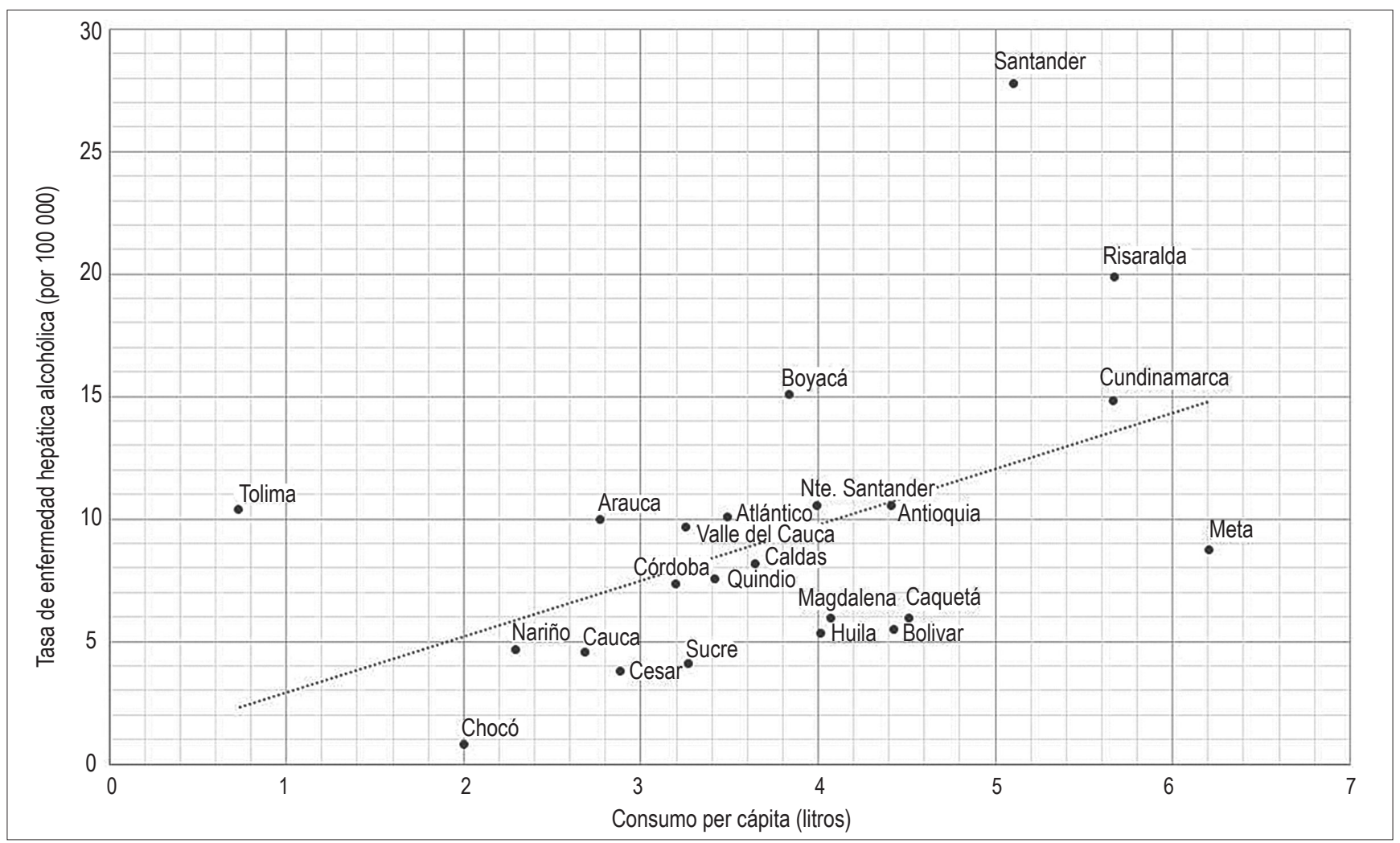

Figura 2. Relación entre las ventas per cápita de alcohol y la tasa estimada de enfermedad hepática alcohólica por departamento en el año 2012. 
nuestros estimados, sino para el diseño de intervenciones de salud pública que enfrenten este problema social.

\section{Agradecimientos}

Este trabajo recibió el apoyo de la Facultad de Medicina de la Pontificia Universidad Javeriana. Los autores declaran no tener ningún conflicto de interés.

\section{REFERENCIAS}

1. Reuben A. Pearls of pathology. Hepatology. 2003;37(3):715-8.

2. Roman S, Zepeda-Carrillo EA, Moreno-Luna LE, Panduro A. Alcoholism and liver disease in Mexico: genetic and environmental factors. World J Gastroenterol. 2013;19(44):7972-82.

3. Sandoval Ferrer JE, Lanigan Gutiérrez ME, Gutiérrez Chapman L. Conocimientos y actitudes de la población acerca del alcohol y el alcoholismo. Rev Cuba Med Gen Integr. 1999; 16(1):13-7.

4. Torres de Galvis Y. Alcohol: Prevalencia de consumo y dependencia en Colombia. CES Med. 2009;14(1): 60-72.

5. Londoño Pérez C, García Hernández W, Valencia Lara SC, Vinaccia Alpi S. Expectativas frente al consumo de alcohol en jóvenes universitarios colombianos. An Psicol. 2005;21(2):259-67.

6. Giraldo Montoya AM, Barraza Amador M, Villa Velásquez H, Martínez JW, García Castro G. Caracterización epidemiológica de pacientes con cirrosis en una consulta de gastroenterología en Pereira, Colombia, 2009-2012. Rev Med Risaralda. 2014;20(2):86-94.
7. Idrovo, V. Cirrosis y bebidas alcohólicas artesanales. Rev Col Gastroenterol 2007;22(2):82.

8. Mendez-Ayala A, Nariño D, Rosselli D. Burden of epilepsy in Colombia. Neuroepidemiology. 2015;44(3):144-48.

9. Rosselli D, Rueda JD. Burden of pneumococcal infection in adults in Colombia. J Infect Public Health. 2012;5(5):354-9.

10. Borja-Aburto VH. Estudios ecológicos. Salud Pública Mex. 2000;45(6):533-8. Disponible en: http://www.scielosp. $\mathrm{org} / \mathrm{pdf} / \mathrm{spm} / \mathrm{v} 42 \mathrm{n} 6 / 3979$

11. Valera Antequera D, Pacheco García OE, Huguett Aragón CM, Solarte Agredo IN. Viabilidad y factibilidad del uso de los RIPS como fuente de información para la vigilancia en salud. Instituto Nacional de Salud 2012. Disponible en: http://aulavirtual.ins.gov.co/ins_gea_cursos/material_ consulta/biblioteca/Viabilidad y Factibilidad de uso de_RIPS_en_vigilancia_en_salud_p\%C3\%BAblica.pdf.

12. Schwartz JM, Reinus JF. Prevalence and natural history of alcoholic liver disease. Clin Liver Dis. 2012;16(4):659-66.

13. Williams JA, Manley S, Ding WX. New advances in molecular mechanisms and emerging therapeutic targets in alcoholic liver diseases. World J Gastroenterol. 2014 Sep 28;20(36):12908-33.

14. Shukla SD, Lim RW. Epigenetic effects of ethanol on the liver and gastrointestinal system. Alcohol Res. 2013;35(1):47-55.

15. Torruellas C1, French SW1, Medici V1. Diagnosis of alcoholic liver disease. World J Gastroenterol. 2014;20(33):11684-99.

16. Profamilia. Encuesta nacional de demografía y salud: Colombia 2012. Disponible en: http://www.profamilia. org.co/encuestas/Profamilia/Profamilia/index.php?optio $\mathrm{n}=$ com_content\&view $=$ article $\& \mathrm{id}=62 \&$ Itemid $=9$

17. Euromonitor International. Alcohol ilegal en seis países de América Latina. Disponible en: http://www.euromonitor.com/ medialibrary/PDF/140410SABMillerAIPPTLatAm.pdf 